\title{
LA EDUCACIÓN MUSICAL COMO HERRAMIENTA PARA EL DESARROLLO DE DESTREZAS Y HABILIDADES EN LOS ESTUDIANTES
}

Music education as a tool for the development of artfulness and abilities in students

Floralba del Rocío Aguilar Gordon

Universidad Politécnica Salesiana del Ecuador

〈faguilar@ups.edu.ec〉

Daniela Alejandra Villacís Marín

Orquesta Sinfónica Nacional del Ecuador

«villacisdaniela123@gmail.com»

Steven Marco Narváez Flores

Gobierno Autónomo Descentralizado Municipal de Rumiñahui.

«stevennarvaez@hotmail.com» 

en los estudiantes

\section{Resumen:}

El presente documento nace de la desventaja encontrada en el plan nacional de estudios secundarios propuesto en el Ecuador de la última década a propósito del retiro de la asignatura de educación musical, misma que pasó a formar parte de la abarcadora educación cultural y artística. La desventaja parte del desconocimiento y la poca consciencia acerca de la importancia de la música en el contexto ecuatoriano. El objetivo de este artículo es reflexionar acerca de las ventajas de la educación musical para el desarrollo de destrezas y habilidades en los estudiantes ecuatorianos del nivel secundario. La principal aportación del documento es el análisis comparativo de los currículos de educación en otros países que permite tener una idea clara de la realidad que circunda el tema en otras latitudes. La metodología utilizada en la estructuración de este trabajo es de carácter inductiva - deductiva, se apoya en la investigación bibliográfica y se auxilia en métodos filosóficos como el fenomenológico y el hermenéutico. Este manuscrito contiene una aproximación conceptual sobre la realidad curricular ecuatoriana, presenta brevemente algunos modelos educativos musicales, reflexiona acerca de la educación musical como referente para el desarrollo de destrezas y habilidades que aportan a la formación integral del ser humano y finalmente, propone algunas perspectivas y desafíos de la educación musical.

Palabras clave: Educación musical, currículo nacional, destrezas, habilidades, estrategia.

\section{Abstract:}

This document is born out of the disadvantage found in the national plan of secondary studies proposed in Ecuador in the last decade regarding the withdrawal of the subject of music education, which became part of the comprehensive cultural and artistic education. The disadvantage comes from ignorance and lack of awareness about the importance of music in the Ecuadorian context. The objective of this article is to reflect on the advantages of music education for the development of skills and abilities in Ecuadorian high school students. The main contribution of the document is the comparative analysis of education curricula in other countries that allows to have a clear idea of the reality that surrounds the subject in other latitudes. The methodology used in the structuring of this work is of an inductive - deductive nature, is supported by bibliographical research and is supported by philosophical methods such as the phenomenological and the hermeneutic. This manuscript contains a conceptual approach to the Ecuadorian curricular reality, briefly presents some musical educational models, reflects on musical education as a reference for the development of skills and abilities that contribute to the integral formation of the human being and finally, proposes some perspectives and challenges of music education.

Keywords: Music education, national curriculum, artfulness, abilities, strategy. 

en los estudiantes

\section{Introducción}

El ensayo académico presenta la realidad incongruente en la que se ha enmarcado a la educación musical con las modificaciones superficiales dadas en el Currículo de Educación del Ecuador desde el año 2008, reflexiona acerca de las desventajas que trae consigo la falta de Educación Musical con peso y valor académico e invita a repensar acerca de esta problemática en el Ecuador.

Es de conocimiento general que, en el año 2009 mediante el acuerdo ministerial Nro. 0611-09 establecido en el Currículo de EGB y BGU (2008), se pone en práctica un renovado plan curricular a impartirse de manera general en los establecimientos educativos a nivel nacional.

Con este antecedente, la asignatura anteriormente llamada educación musical, pasa a formar parte de una idea más abarcadora llamada educación cultural y artística, la cual se mantiene con el currículo de cultura estética planteada en 1996, dentro del acuerdo se aclara que, "para el desarrollo de este documento se partió de los principios de la pedagogía crítica, considerándose que el estudiante debía convertirse en el principal protagonista de los procesos de enseñanza y aprendizaje" (Educación Cultural y artística, 2008, 7). Este nuevo enfoque pretende contribuir en la capacidad de los individuos para resolver problemas cotidianos de forma solidaria, ética e innovadora, con el fin de crear una sociedad íntegra en todos sus sentidos.

Al establecer la asignatura de educación cultural y artística como directriz para las diferentes ramas artísticas -danza, teatro, dibujo, música- en el medio social, se deja en segundo plano la educación musical y se la plantea como una materia especial u opcional. Con el pasar de los años, este planteamiento curricular provocó un notable problema, el cual se formula como la ausencia de consciencia social en torno a los beneficios de la música en el desarrollo integral de los estudiantes.

La música parte de una serie de estímulos donde se recepta, se analiza, se estructura y se procesa información visual, auditiva y emotiva aludiendo a las sensaciones del receptor, evidentemente este proceso desarrolla y potencia destrezas y habilidades. La música proporciona una serie de emociones y sensaciones en el ser humano y por ello se convierte en un factor de vital importancia para el proceso enseñanza-aprendizaje. Es menester cuestionarse acerca de la fuente generadora de la música y de sus diferentes manifestaciones (emoción, sensación,). En este sentido, Díaz (2010) postula que:

... la emoción musical probablemente emerge de la activación inicial de regiones cerebrales directamente involucradas en la percepción musical y de la activación subsecuente de sistemas emocionales ligados a la percepción sensorial, además de la activación coherente de zonas de la corteza cerebral involucradas en la extracción de significado musical (547).

Es decir, la música y las sensaciones que ésta produce pueden generar en un solo momento mayor actividad cerebral, misma que aparte de distintas ventajas terapéuticas, de lenguaje, memoria, conducta, entre otras, permite que el estudiante sea capaz de adquirir nuevos conocimientos de manera divertida, trascendental e innovadora.

En palabras de Vernia, A., Gustems, J. y Calderón, C. (2016), el primero en preocuparse de mostrar los elementos corporales que inciden en la enseñanza-aprendizaje del lenguaje musical, teniendo en cuenta sus contenidos rítmicos fue Jacques Dalcroze quien trató de vincular la enseñanza del lenguaje musical a la educación rítmica y se fue el primero en "organizar un método de enseñanza del lenguaje musical a partir de las destrezas rítmicas, mediante series de ejercicios diseñados con este fin" (1) que evidencian cómo la educación musical proporciona un desarrollo de ambos hemisferios cerebrales, razón por la cual, algunos estudiosos recomiendan la integración de la enseñanza de música desde la educación básica. 

en los estudiantes

Desde esta perspectiva, estudiosos como Domingo-Cebrián (2008) sostienen que, con la música como disciplina, se consigue un aumento de las capacidades cerebrales de la siguiente forma:

Hemisferio izquierdo: Percepción rítmica, control motor, rige mecanismos de ejecución musical, el canto, aspectos técnicos musicales, lógica y razonamiento, captación de lo denotativo, percepción lineal. Hemisferio derecho: Percepción y ejecución musical, creatividad artística y fantasía, captación de la entonación cantada, percepción visual y auditiva, percepción melódica y del timbre, expresión musical, apreciación musical (2).

La práctica de la música favorece el desarrollo cerebral y nervioso en su totalidad puesto que la educación musical al desarrollar las posibilidades de nuestros circuitos neuromusculares permite en palabras de Domingo-Cebrián (2008) cultivar el sistema nervioso, trabajar nuestro desarrollo en general, nuestros estados afectivos, nuestra receptividad, nuestra atención, etc., de manera que "la educación musical estimula todas las facultades del ser humano: abstracción, razonamiento lógico y matemático, imaginación, memoria, orden, creatividad, comunicación y perfeccionamiento de los sentidos" (3).

La música, el pensamiento, la expresión, el movimiento y la acción se encuentran vinculados, pues, el cuerpo, la palabra, los objetos y el contexto contienen en sí mismos algunas dimensiones sonoras que permiten producir nuevos conocimientos y desarrollar capacidades mentales diversas como las sugeridas por Domingo-Cebrián (2008): "análisis, abstracción, expresión, simbolización, síntesis y atención, favoreciendo la imaginación y la creatividad" (2) que de alguna manera fortalecen los procesos de adquisición y desarrollo de los aprendizajes básicos de la lectura, la escritura y el cálculo.

En este contexto, el objetivo del presente documento es exponer el plus educativo que proporciona la educación musical como medio potenciador de destrezas y habilidades en el desarrollo integral de los estudiantes mediante la comparación de realidades internacionales.

El presente documento se direccionó a través del uso de una investigación cualitativa en la cual se usó una técnica teórico-comparativa basada en la observación y recolección de datos, para interpretar y comparar el fenómeno social educativo respecto a la educación musical. Este sistema comparativo se lleva a cabo al referenciar diferentes sistemas musicales utilizados en España, Venezuela y Puerto Rico ya que el carácter curricular de dichos países muestra un gran apoyo a la inclusión de la educación musical como herramienta para el desarrollo integral de los estudiantes.

Esta línea de argumentación podría acompañar a los aportes de Blacking (2003) quien, al referirse a la inclusión de la educación musical en el desarrollo de los estudiantes, establece que las funciones de la música tienen como ente y factor decisivo el promover un talento musical de tal manera que, el desarrollo de esta inteligencia influye en la elección y en la producción de conceptos culturales y materiales que constituyen una sociedad o una identidad determinada.

Este manuscrito se encuentra estructurado en cuatro apartados que permiten repensar en la importancia de la música en el currículo educativo y en el desarrollo integral del ser humano, los componentes que guían la comprensión de la temática son los siguientes: aproximación conceptual a la realidad curricular ecuatoriana; análisis de modelos educativos musicales; la música como referente para el desarrollo de destrezas y habilidades; y, finalmente, perspectivas y desafíos de la educación musical.

\section{Aproximación conceptual a la realidad curricular ecuatoriana.}

A partir de la última reforma realizada en el currículo del Ecuador se han suscitado varios cambios como el surgimiento de criterios de evaluación a los participantes del proceso 

en los estudiantes

educativo, se transforman los enfoques y tendencias pedagógicas en la educación del Ecuador. Uno de los objetivos de la nueva reforma educativa ecuatoriana es la formación de un individuo justo, innovador, solidario y capaz de resolver problemas cotidianos gracias a la instrucción recibida a lo largo de su formación educativa, tal como se encuentra establecido en el perfil de salida propuesto en el currículo 2016, "en el currículo se plasman en mayor o menor medida las intenciones educativas del país, se señalan las pautas de acción u orientaciones sobre cómo proceder para hacer realidad estas intenciones y comprobar que efectivamente se han alcanzado" (Currículo De Los Niveles De Educación Obligatoria, 2016, 4).

El currículo nacional de los últimos tiempos, a partir de 2010 se ha orientado hacia el desarrollo de destrezas con criterio de desempeño que el individuo debe aplicar para la resolución de problemas cotidianos de la realidad en la que vive. Esto obliga a la educación a proporcionar conocimientos inter y multidisciplinarios que aporten para la adaptabilidad de los conocimientos del estudiante frente a situaciones concretas. El sistema educativo nacional basado en la flexibilidad del currículo se propone respetar la individualidad de aprendizaje y fomentar las actividades en equipo y el desarrollo de las principales operaciones mentales ${ }^{1}$ del sujeto como razonar, explicar, crear, etc.

Además, se menciona que cada institución debe tomar en cuenta la diversidad de la comunidad educativa, las necesidades especiales, entre otros aspectos, para ofertar algún tipo de área adicional al currículo establecido, siempre que esté aprobada por las autoridades pertinentes y se realice con fondos de la institución misma, aspecto que se sustenta en la siguiente cita referida a la autonomía institucional: "Las instituciones educativas disponen de autonomía pedagógica y organizativa para el desarrollo y concreción del currículo, la adaptación a las necesidades de los estudiantes y a las características específicas de su contexto social y cultural" (Currículo De Los Niveles De Educación Obligatoria, 2016, 15).

En cuanto al currículo de educación cultural y artística (2008) en el currículo nacional se expresa que:

La cultura y las artes desempeñan un papel fundamental en la vida de las personas y, como tales, promueven experiencias y aprendizajes básicos para todos los ciudadanos. Son un recurso privilegiado a la hora de descubrir quiénes somos y cómo nos relacionamos, posibilitando formas de pensamiento tan rigurosas como las de las ciencias o las matemáticas, y tan divergentes como las de la filosofía o la literatura. Asimismo, contribuyen a que nuestras vidas sean más plenas en todos los sentidos, generando una parte significativa del capital intelectual y creativo, personal y social (50).

Es posible considerar una incongruencia entre discurso y acción en cuanto al primer párrafo de la sección de educación cultural y artística del currículo citado anteriormente, en donde se resalta en gran manera la excelente función de las artes en la vida como tal, sin embargo, hay que mencionar que la carga horaria que se le designa a ésta cátedra en la educación nacional es casi nula (tal como se demuestra en la Tabla 1) dejando mucho que desear principalmente a los profesionales en una o varias ramas de las artes, puesto que

\footnotetext{
${ }^{1}$ Las operaciones mentales son procedimientos lógicos que realiza el ser humano con la finalidad de enfrentarse, organizar, analizar, reflexionar, sintetizar, procesar y sistematizar la información conforme a las necesidades, experiencias y conocimientos previos (Aguilar, 2017, 47). A su vez las operaciones mentales generan productos que emergen de los procesos psicológicos: motivación, atención, intención, memoria, inteligencia, imaginación, creatividad y es necesario considerar que las acciones humanas dependen de estos procesos y operaciones (Aguilar, 2017, 53). La didáctica musical resulta de vital importancia para el desarrollo de este tipo de operaciones fundamentales en el ser humano.
}

Universidad UTE / CC BY Tsafiqui, Revista de Investigación Científica, № 112018 
Aguilar Gordon Floralba del Rocío/ Villacís Marín Daniela Alejandra/ Narváez Flores Steven La educación musical como herramienta para el desarrollo de destrezas y habilidades en los estudiantes

conocen los beneficios que éstas otorgan en el desarrollo integral no solo de determinada comunidad educativa sino de cualquier ser humano que experimente una educación artística.

Tabla 1: Carga horaria de la asignatura de Educación Cultural y Artística en Educación General Básica y en Bachillerato Unificado.

\section{a. Educación General Básica}

\begin{tabular}{|c|c|c|}
\hline \multicolumn{2}{|c|}{ Subnivel de Básica Preparatoria } \\
\hline Áreas & Asignaturas & Carga horaria \\
\hline Currículo Integrador por ámbitos de aprendizaje & 25 \\
\hline ducación Cultural y Artística & Educación Cultural y Artística & 3 \\
\hline Educación Física & Educación Física & 5 \\
\hline \multicolumn{2}{|c|}{ Proyectos Escolares } & 2 \\
\hline \multicolumn{2}{|c|}{ Horas pedagógicas totales } \\
\hline
\end{tabular}

Fuente: Currículo Nacional, 2008, Ecuador

b. Bachillerato General Unificado

\begin{tabular}{|l|l|c|c|c|c|}
\hline \multirow{3}{*}{$\begin{array}{l}\text { Tronco } \\
\text { común }\end{array}$} & \multicolumn{1}{|c|}{ Áreas } & \multicolumn{3}{|c|}{ Cursos } \\
\cline { 2 - 6 } & Matemática & Matemática & 1 & 2 & 3 \\
\cline { 2 - 6 } & Ciencias Naturales & Física & 5 & 4 & 3 \\
\cline { 3 - 6 } & & Química & 3 & 3 & 2 \\
\cline { 3 - 6 } & Ciencias Sociales & Biología & 2 & 3 & 2 \\
\cline { 3 - 6 } & Historia & 2 & 2 & 2 \\
\cline { 3 - 6 } & Educación para la & 2 & 2 & - \\
\cline { 3 - 6 } & ciudadanía & & & \\
\cline { 3 - 6 } & Lengua y Literatura & Lengua y Literatura & 5 & 5 & 2 \\
\hline & Lengua Extranjera & Inglés & 5 & 5 & 3 \\
\hline & Educación Cultural y & Educación Cultural y & 2 & 2 & - \\
& Artística & Artística & & & \\
\cline { 2 - 6 } & Educación Física & Educación Física & 2 & 2 & 2 \\
\hline
\end{tabular}


Aguilar Gordon Floralba del Rocío/ Villacís Marín Daniela Alejandra/ Narváez Flores Steven La educación musical como herramienta para el desarrollo de destrezas y habilidades en los estudiantes

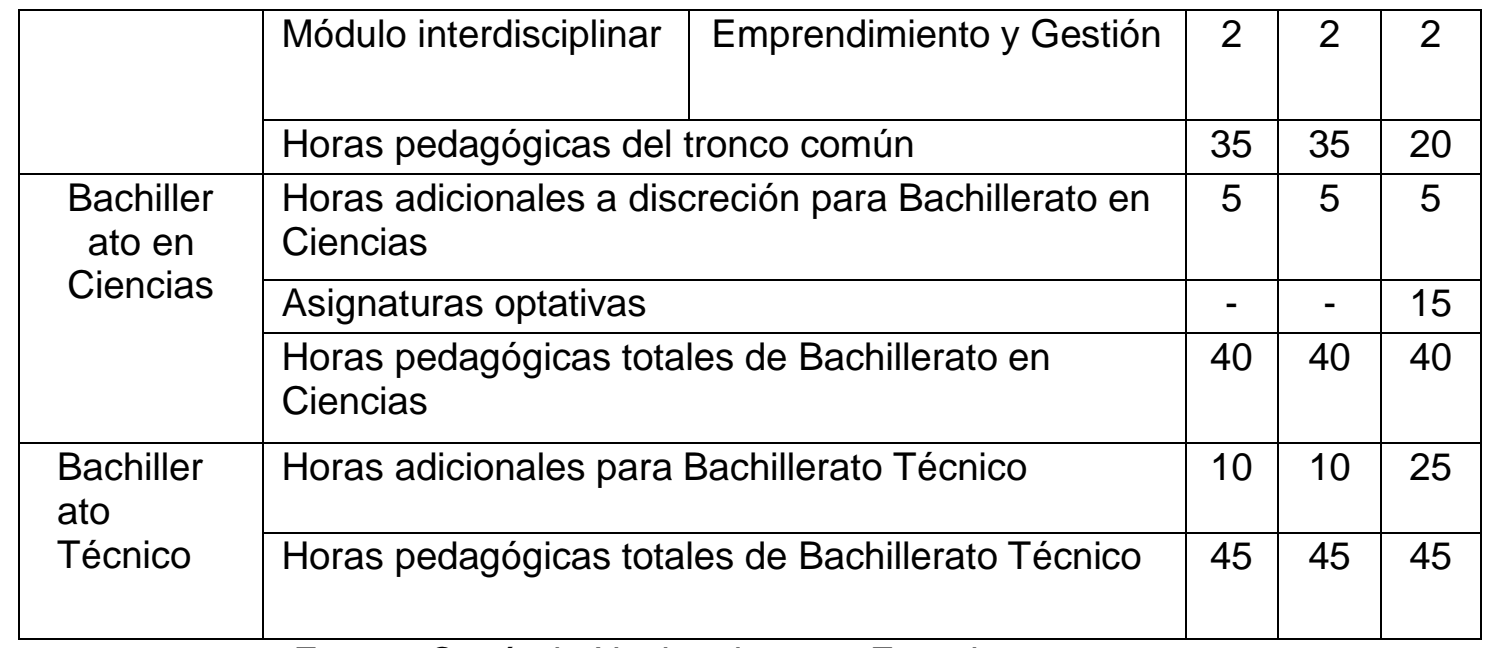

Fuente: Currículo Nacional, 2008, Ecuador

La música es la rama del arte con la que todas las personas están relacionadas, "escuchamos música desde la cuna o, incluso, en el período de gestación. Los bebés, en los primeros meses de vida, tienen la capacidad de responder a melodías antes que a una comunicación verbal de sus padres" (Manes, 2015, 2), mucho más quienes se encuentran en proceso de formación y están atentos a todo lo que sucede a su alrededor y se encuentran estimulados a aprender sobre todo en cuanto observan, incluso sin necesidad de recibir la información de un profesional.

Por ejemplo, la educación impartida por Finlandia tiene el compromiso de evocar una enseñanza lúdica y que se aprenda a partir de sus propios conocimientos, evitando el hostigamiento conceptual del que vemos implementado ya varios años en nuestro país "Cada día es dedicado a una disciplina (música, deporte, actividades manuales o artísticas, lengua materna, matemáticas) pero los niños trabajan solamente durante la mañana y siempre de manera muy atractiva. La tarde es reservada al juego" (Robert, 2006, 3), el enfoque que se da a la pedagogía en este país puede parecer extraña, sin embargo, la innovación que demuestra esta propuesta y aún más importante, sus resultados, han posicionado a la educación de Finlandia como la primera a nivel mundial.

Las competencias, destrezas y habilidades que se pueden obtener y desarrollar gracias a la adecuada aplicación de la didáctica musical en el aula son mayores a las que se puede obtener sin su aplicación, la didáctica musical trae consigo una serie de ventajas e incluso se convierte en un recurso facilitador que permite a los docentes avanzar el tratamiento de los contenidos con mayor comodidad. La didáctica musical despierta en el ser humano una serie de emociones, propicia el desarrollo de diferentes procesos cognitivos y activa los órganos sensoriales que emiten impresiones y sensaciones diferentes en cada uno de los sujetos. Es así como:

la emoción musical, en paralelo con otras emociones y procesos cognitivos superiores, se integra progresivamente a lo largo de los relevos de la vía auditiva pero que no termina en las zonas de recepción primaria de la sensación sonora ni en la activación de las estructuras subcorticales del sistema límbico, sino que el estímulo es finalmente procesado mediante una dinámica intermodular en áreas extensas del cerebro para integrar la experiencia musical afectiva y consciente" (Díaz, 2010, 550).

Además, de lo expresado y de acuerdo con la cita precedente se entiende que la música proporciona un alto nivel de desarrollo de componentes cerebrales que permite ampliar el abanico de destrezas que un estudiante puede adquirir u optimizar durante su formación educativa. 

en los estudiantes

Generar un ambiente adecuado para el aprendizaje es un tema común entre profesionales pedagogos, es decir, lograr que los estudiantes se sientan cómodos y tranquilos mediante el acercamiento directo e indirecto a sus emociones y distintas personalidades, haciendo uso de distintas estrategias. Un estudiante que no está a gusto en el aula de clase no es una mente abierta dispuesta a adquirir conocimientos y habilidades con facilidad a pesar de que el docente aplique varias técnicas para alcanzar sus objetivos, pues una mente estresada, incómoda, aturdida, etc., no es un campo fácil para ningún maestro, sin embargo:

...está demostrado que los conocimientos, habilidades, destrezas, hábitos, actitudes y formas de pensar de las personas se pueden modificar y de hecho son susceptibles de verse influidos fuertemente por variables contextuales, del ambiente, de la sociedad y del sistema educativo, pudiendo ser modificadas y mejoradas. Además, y tan importantes como las anteriores, deben mencionarse las variables afectivas y motivacionales, que también afectan de manera relevante el desarrollo de las habilidades cognitivas y el aprendizaje (Schmidt, 2006, 2).

La música tiene un doble compromiso con la educación ya que funciona como un potenciador de destrezas y habilidades permitiendo llegar a las emociones de los estudiantes. La música es una construcción humana de sonidos coordinados y encauzados que, auxiliada en instrumentos y con una expresión motora optimizada, "se constituye en un estímulo sonoro espaciotemporalmente organizado que resulta en una percepción auditiva compleja al estar dotada de estados emocionales y figurativos conscientes estéticamente significativos y culturalmente valorados" (Díaz, 2010, 543). Se puede decir que la estimulación mediante la didáctica musical es sencilla y resulta fácil de aplicar, es mucho más sencilla que un problema matemático o una lectura extensa para analizar. Una canción o un ejercicio rítmico llaman la atención de los estudiantes e incluso los motivan a improvisar melodías hermosas, que bien pueden ser utilizadas como estrategias didácticas ${ }^{2}$ en las distintas áreas del saber.

Otro enfoque en torno a los beneficios de la música se puede basar en los aportes de Uribe (2016) quien plantea que la música interviene a nivel neuroquímico, donde se ha hallado que puede activar o suprimir sustancias químicas que van debido a específicas tonalidades con repercusiones en el sistema inmunológico y hormonal; y por ende estas reacciones repercutirán a nivel cognoscitivo de manera positiva. Lo anterior demuestra que es importante tomar en cuenta los beneficios y las posibilidades innovadoras que proporciona la música en varios aspectos, especialmente en el ámbito educativo.

Cabe recordar que cada persona posee características únicas que varían conforme al contexto sociohistórico y cultural en que se encuentra el sujeto. La calidad de los aprendizajes depende del estilo, del tipo de inteligencia, de la diversidad de creencias, gustos y preferencias de cada uno de los seres humanos. Los tipos de pensamiento, las inteligencias múltiples, la inteligencia emocional son una realidad única y diversa a la vez en la medida en que todos los seres humanos los poseen, pero el grado de desarrollo es diferente en cada uno de ellos dependiendo de la variedad de factores endógenos y exógenos que operan en el sujeto.

En este contexto, a decir de Aguilar (2016) es indispensable que el docente conozca a plenitud los estilos de aprendizaje de sus estudiantes ${ }^{3}$, comprenda "la forma en que sus

\footnotetext{
${ }^{2}$ Una estrategia didáctica es concebida como estructura de actividad, en la que se hace real los objetivos y contenidos, se entiende como un conjunto de decisiones que pretende alcanzar objetivos reales, procedimientos, recursos y contenidos, también se suele considerar como un plan de acción que incluye tareas o actuaciones que pone en marcha el docente de manera sistemática para que los estudiantes alcancen los objetivos de aprendizaje previstos y los contenidos seleccionados (Aguilar, 2016, 171).

${ }^{3}$ Los estilos de aprendizaje involucran algunos componentes fundamentales que permiten comprender el desenvolvimiento humano en su totalidad. Un estilo de aprendizaje involucra a todo un conjunto de características fisiológicas, pedagógicas, contextuales-histórico-socio-culturales, cognitivas,
} Universidad UTE / CC BY Tsafiqui, Revista de Investigación Científica, № 112018 

en los estudiantes

estudiantes logran procesar la información, comprender, asimilar, estructurar y aplicar los contenidos, la manera en que forman y utilizan conceptos, el modo en que interpretan la información, resuelven los problemas, seleccionan los medios de representación" (168), con la finalidad de generar mecanismos para la construcción del conocimiento y para alcanzar aprendizajes significativos. El conocimiento del estudiante por parte del docente permitirá que éste utilice métodos, técnicas, estrategias y procedimientos adecuados para llevar adelante el proceso formativo que independientemente del área curricular y de los contenidos científicos que se estudien podrá emplear a la didáctica musical como la principal herramienta para el cumplimiento de los resultados de aprendizaje y para el desarrollo de competencias, habilidades y destrezas en el estudiante. Esto no significa que el aprendizaje basado en la didáctica musical sea exclusivo de quienes tienen más desarrollada la inteligencia musical, pues todas las personas cantan y bailan una multiplicidad de ritmos conforme al contexto en el que se encuentran, así:

Las personas cantan y bailan juntas en todas las culturas. Sabemos que lo hacemos hoy y lo seguiremos haciendo en el futuro. Podemos imaginar que lo hacían también nuestros ancestros, alrededor del fuego, hace miles de años. Somos lo que somos con la música y por la música, ni más ni menos (Manes, 2015, 3).

\section{Análisis de modelos educativos musicales}

Es significativa la importancia que tiene la música en el desarrollo integral de un ser humano, tal es su impacto que instituciones de varios países como: España, Venezuela, Puerto Rico, naciones en las que por décadas vienen desarrollando sus propios modelos educativos en los que se prioriza las materias artísticas en sus pensum de estudios. A continuación, se hace referencia a los modelos educativos mencionados tomando en cuenta únicamente los aspectos relacionados a la educación musical para de esta manera llegar al cometido del presente documento.

En España la realidad curricular en torno a la música no es tan distinta a la de Ecuador -hablando teóricamente- En la LOCE (Ley Orgánica de la Calidad de la Educación) la música está definida en un área más específica a la expresión artística y a la creatividad, esta realidad esta expresada en la tesis doctoral de Lotova (2008) misma que expresa: la música está comprendida en el área de Comunicación y representación, puntualmente plantea "la música es expresión y también comunicación. Percibirla conscientemente y utilizarla como tal equivale a lo que en el lenguaje ordinario significa hablar y comprender" (73). Así, como en Ecuador la materia musical no está explicita dentro de los currículos académicos más bien es implícita y forma parte de un área artística.

A diferencia de Ecuador, en España se tiene bien planteado los contenidos conceptuales, procedimentales y de actitud en tanto a la educación musical. Según los aportes de Lotova $(2008,74)$ la educación musical sintetiza 6 apartados en función del currículo educativo actual en España:

1.- Ruido, silencio y música. Busca la discriminación auditiva mediante la familiarización del niño con su entorno sonoro sin necesidad de una materialización teórica.

procedimentales, afectivas, actitudinales que se evidencian en el instante en el que una persona debe enfrentar una situación de aprendizaje; implica distintas formas en que el sujeto cognoscente puede aprender; conlleva todo un conjunto de herramientas, métodos, técnicas y procedimiento, actitudes, aptitudes y acciones que emplea una persona en el proceso de aprendizaje y de construcción del conocimiento (Aguilar, 2016, 167-168). 

en los estudiantes

2.- La expresión vocal y canto. Tiene por objeto, presentar una cierta complejidad al interpretar una canción mediante la voz, de esta forma potencia la capacidad comunicativa del estudiante.

3.- Las propiedades sonoras del cuerpo. Este apartado tiene como fin la expresión y la comprensión de sentimientos y emociones mediante la experimentación sonora tanto con el cuerpo como con los objetos del entorno.

4.- Audiciones musicales. De esta manera se busca establecer una mayor atención ante algún tema o ambiente educativo y de la misma forma enriquece la cultura musical del estudiante.

5.- El movimiento rítmico y la danza. Busca el desarrollo motriz, potencia las relaciones interpersonales y evalúa la capacidad de reaccionar físicamente ante la música.

6.- Cultura musical. Mediante la interpretación de canciones, obras, bailes y danzas folclóricas plantean acercar al niño con la cultura de su región o de otras procedencias.

Es interesante presentar estos apartados, mismos que denotan la importancia hacia la música y principalmente, expresan una relación estrecha con el desarrollo de otras destrezas y especifican las ventajas que estas traen al contexto educativo. Para reforzar este hecho vale referir al pensamiento de Duran y Castell (2012) quienes sostienen:

...consideramos la música como un elemento educativo que incide en el desarrollo de determinadas capacidades físicas y psíquicas del individuo, lo enriquece y le suministra instrumentos para que se realice como ser humano en un contexto social y cultural concreto, entenderemos por qué también los pedagogos han estudiado la incidencia del aprendizaje musical en el desarrollo integral de la persona (184).

Otro ejemplo, de la materia musical implementada en un currículo educativo es en Venezuela -obviando en este caso la situación actual social y económica del país- este país tiene un acervo musical muy amplio, en sus mejores épocas era un referente innato de confluencia artística en todos los ámbitos y géneros musicales, lo que interesa abordar en este momento es la estructura y planteamiento de la música en el ámbito educativo. Para un acercamiento a esta realidad es preciso referenciar las contribuciones realizadas por Bordones (2013) quien aclara que:

....el Ministerio del Poder Popular para la Educación creó, en el año 2007, el nuevo Subsistema de Educación Inicial Bolivariana, el cual comprende niños y niñas entre cero (0) y seis (6) año (...) en el Currículo del Subsistema de Educación Primaria Bolivariana en su Currículo Básico Nacional (CBN), garantiza la formación integral de los niños y las niñas desde los seis (6) hasta los (12) años de edad o hasta su ingreso al subsistema siguiente" (pp. 125-126)

A partir de estos diseños provenientes del Ministerio del Poder Popular para la Educación y refiriéndose al CBN, son planteados 6 grados educativos con sus respectivas directrices. De esta manera Bordones (2013, pp. 126-127) resalta los contenidos a tratarse en cada grado, en este caso enfatizando los musicales:

1er grado. Repertorio de canciones venezolanas; creación de producciones artísticas a través de la música y la danza; representación de la danza a través de la música tradicional; sonorización con instrumentos y onomatopeyas.

2do grado. Expresión a través de la música y la pintura como manifestaciones artísticas; vocalización y entonación del Himno Nacional, regional, institucional y otros; ejecución de 

en los estudiantes

instrumentos musicales; improvisación de producciones artísticas a través de la música; interpretación de instrumentos musicales.

3er grado. Identificación del sonido y el ritmo; valoración de la música y la pintura como manifestaciones artísticas; ejecución instrumentos musicales; la música, ritmos venezolanos, africanos, europeos y caribeños; entonación del Himno Nacional; conocimiento de la expresión musical: tiempo y silencio en la música

4to grado. Caracterización del espacio, tiempo, sonido, ritmo y movimientos en manifestaciones artísticas; manifestación de sentimientos, emoción y afianzamiento de la identidad venezolana en expresiones artísticas: la música, ritmos venezolanos,

5to grado. Clasificación de los instrumentos musicales según su origen; estudio del timbre de voz en la formación en la formación de coros; diferenciación de los sonidos, la contaminación sónica ejecución de instrumentos musicales; entonación del Himno Nacional, regional, institucional y otros himnos alusivos a diferentes efemérides; representación creativa de una melodía.

6to grado. Afianzamiento de la expresión musical en base a la diferenciación de los instrumentos musicales según su origen, el timbre, los sonidos, la contaminación sónica, ritmos regionales. Entonación del Himno Nacional, Himno del estado, Himno de la escuela. Importancia y significado de las canciones folklóricas y tradicionales venezolanas.

Es notoria la aplicación e importancia de la música que se plantea en el CBN de Venezuela ya que parten de conocimientos tradicionales para lograr fortalecer el desarrollo integral del estudiante donde el profesor de cultura estética debe estar pendiente del cumplimiento de estos contenidos; pero no todo es bueno, Bordones (2013) acota una debilidad encontrada en este sistema:

...el docente integral que no posea conocimientos musicales jamás podrá impartir estos conocimientos, tan importantes para el desarrollo integral. De aquí nace la importancia de que sea un docente en música el que desarrolle estos contenidos, donde el profesor se vea en la necesidad de elaborar su planificación orientada a la enseñanza de los elementos constitutivos de la música y que se ven plasmados en el CBN (129).

Como último referente para la argumentación de este documento, se realizó un análisis al sistema educativo en Puerto Rico, mismo del que la situación educativa -en tanto a la música- no es muy distinta de los otros países ya abordados; la diferencia de éste es la gran influencia norteamericana que presenta en su currículo educativo. López (2010) asevera que:

Puerto Rico trata de mantener su propia cultura musical y la responsabilidad social de educar musicalmente a sus ciudadanos en edad escolar, aunque lo cierto es que (...) la legislación educativa estadounidense es la que determina las bases y el funcionamiento educativo en la Isla (72-73).

En la Isla, se aplica una ley norteamericana llamada No Child Left Behind Act la cual fue establecida en 2001, incluyó las artes como materia básica del currículo educativo ya que se basó en investigaciones que evidencian las contribuciones de las artes en el desarrollo educativo de otras áreas académicas. Por otra parte, en los años ochenta Estados Unidos genera reformas educativas que se plantean mediante la aprobación de estándares educativos y limitando la flexibilidad del currículo a nivel de educación pública.

López $(2010,75)$ establece que los estándares de contenido y ejecución en torno a la educación musical se miden en 4 niveles de calidad:

\section{$\checkmark$ Primer nivel o aprendiz.}

Universidad UTE / CC BY Tsafiqui, Revista de Investigación Científica, № 112018 

en los estudiantes

\author{
$\checkmark$ Segundo nivel o ejecutante. \\ $\checkmark$ Tercer nivel o creador. \\ $\checkmark$ Cuarto nivel o estudiante artista.
}

Es verdad que, los tres modelos musicales abordados en el presente documento son evidentemente distintos debido a la diversidad cultural, geográfica, social, económica e inclusive histórica de cada uno de los países, pero hay algo en común entre ellos, los insumos teóricos que invitan a tomar conciencia acerca de la importancia de la música en el desarrollo íntegro e integral de un estudiante considerando los beneficios que trae consigo. Esto incita a pensar que si en la educación ecuatoriana de nivel medio, se aplicase algo de las vivencias experimentadas en los modelos de los países analizados ayudaría a mejorar la calidad de educación impartida por los docentes, los aprendizajes de los estudiantes y sobre todo permitiría formar integralmente al ser humano.

\title{
La música como referente para el desarrollo de destrezas y habilidades.
}

Es imprescindible establecer los beneficios que trae consigo la música al desarrollo integral de un estudiante; pero antes hay que referir al pensamiento común de la sociedad ecuatoriana en la que actualmente la música es considerada como pasatiempo, entretenimiento, diversión, etc., sustantivos superficiales y que de modo impreciso definen la importancia de la música en la vida de los seres humanos, mucho más importante si se utiliza como una herramienta estratégica de los docentes para impulsar el desarrollo de las destrezas y habilidades de los estudiantes.

Las personas generalmente relacionan a la música con experiencias caracterizadas por la alegría, tristeza, enfermedad, logros, pobreza, religión, etc., en esta ocasión enfocamos el uso de la música para la adquisición de destrezas que permiten al estudiante obtener los mismos conocimientos planificados por las instituciones y docentes, pero con más agilidad y aceptación en todos los sentidos. Con respecto a esta temática "existe una cantidad considerable de buenas investigaciones que pueden esbozar un discurso coherente en defensa de la educación musical como vehículo idóneo hacia una educación integral" (López, $2010,23)$. Por lo que oficialmente, existe respaldo que promueve la valoración de las artes y la música en especial como parte de la educación general obligatoria.

Hablando específicamente de las destrezas que se pueden desarrollar mediante la aplicación de la música como estrategia dentro del aula podemos mencionar la habilidad lingüística o de aprender una lengua extranjera. Por ejemplo, utilizar estímulos como melodías permitiría el desarrollo de los "tres subcomponentes de la aptitud lingüística, el aumento de la habilidad auditiva, un reforzamiento de la habilidad lingüística con el estudio de la letra de las canciones, además de facilitar la memorización al fusionarse elementos sonoros con lingüísticos" (Toscano y Fonseca, 2012, 200).

Estas habilidades pueden no ser específicamente para la lengua extranjera pues la aptitud lingüística funciona en literatura, la habilidad auditiva es un beneficio útil para toda la vida, el aprender letras de canciones permite ejercitar la memoria, el disfrute de la clase y también motiva a los estudiantes y a los docentes seguir poniendo atención a la clase, en fin, se puede mencionar que existen beneficios en gran cantidad con la aplicación de didáctica musical en la clase de lengua extranjera.

Así mismo, si se considera que una de las materias más importantes, pero una de las asignaturas más evitadas por los estudiantes e incluso por los adultos en general son las matemáticas, entonces si se utiliza la música como una estrategia didáctica para impartir matemáticas con certeza, la situación actual cambiaría, incidiría en el cambio de mentalidad del estudiante y se lograrían aprendizajes significativos de los contenidos de esta. Las 

en los estudiantes

matemáticas son de vital importancia para comprender e insertarse en la realidad cotidiana, permite razonar, comprender y resolver problemas. La relación entre música y matemática es estrecha; la ciencia, el universo, el conocimiento, las matemáticas, literatura, son elementos que poseen un orden estricto, sin embargo, no son primera opción de investigación ya que suelen tener un lenguaje poco común que aleja a las personas que no están específicamente interesadas en ellas. La música también tiene este orden, pero su ventaja es que se encuentra a disposición de todas las personas y es capaz de atrapar su atención de manera inmediata.

La enseñanza de las matemáticas se realiza desde corta edad y es allí cuando el docente debe incorporar estrategias didácticas atractivas para atrapar al estudiante y que mejor hacerlo acudiendo a la música como herramienta fundamental, "las matemáticas que se deben presentar en el aula deben estar relacionadas también con la educación sensorial y del lenguaje, con la psicomotricidad, la plástica, los cuentos, la música, y el conocimiento del medio" (Chao, Vásquez y López. 2015, 1012). Con respecto a esta afirmación, no hay duda de que una herramienta viable para enseñar matemáticas desde temprana edad, misma que permite acercar al niño a sensaciones y conocimientos exactos es, la música.

Ahora bien, otra de las ventajas que tiene la aplicación de la educación musical en el aula, es que funciona como un lazo intercultural, capaz de vincular "mundos" diferentes en un solo pensamiento, una sola motivación y además lograr que éstos se fusionen obteniendo así un ambiente de aprendizaje amplio, normativo y con valores.

Hay que tomar en cuenta que las estrategias a aplicar deben estar debidamente planificadas, "evidentemente, estas actividades deberán estar integradas de manera equitativa en un programa variado, significativo, interesante y diversificado de acuerdo con la edad y demás características del grupo" (Bordones, 2012, 122). Es decir, la aplicación de la música como una estrategia dentro del aula tiene beneficios, sin embargo, si no se aplica con la debida organización puede llegar a ser un distractor en lugar de ayuda para los fines deseados.

Prosiguiendo con el tema que motivó la estructuración de este documento, la música contiene elementos que permiten desarrollar habilidades internas, asumir con destreza y agilidad los contenidos. Al respecto, Pérez (2013) demuestra en su investigación elementos como: el principio de repetición, entendido como la interiorización de un pulso que se repite habitualmente, en la música se refiere al ritmo externo e interno, puede ser reconocido cuando se escucha una canción e inmediatamente se siente la necesidad o el impulso de realizar algún movimiento acorde a la misma; el principio de simultaneidad,

Normalmente, y salvo que nos haya gustado mucho, no solemos encontrar encanto en ver una película 2 veces o en leer el mismo libro 2 veces... En la música encontramos un elemento que no se encuentra en otras artes: la simultaneidad, es decir, el escuchar varias circunstancias al mismo tiempo sin que esto constituya incompatibilidad. En un primer grado encontramos la simultaneidad entre el ritmo y la melodía. En un segundo plano destacaríamos las diferentes introducciones de instrumentos que enriquecen la obra aportando más sonidos armónicos. Y entraríamos en más niveles desarrollando así una inagotable fuente de elementos que el oído no es capaz de analizar en una sola pasada y que por eso capta como nuevos en cada ocasión que los escucha. Podríamos denominar esto casi como una segunda dimensión de la música (Pérez, 2013, 12)

Y la memoria, referida a la memoria rítmica o armónica que por ejemplo se evidencia cuando se recuerda una obra o canción y es posible para la persona que escucha predecir tanto armónica como rítmicamente, esto se da después que la misma es escuchada varias veces ya sea consciente o inconscientemente. En fin, en pocas especificaciones es posible entender las características benefactoras de la música desde distintos puntos de vista que estimulan la actividad cerebral en un nivel mayor que sin ella.

Universidad UTE / CC BY Tsafiqui, Revista de Investigación Científica, № 112018 

en los estudiantes

Ha sido histórica la lucha que han enfrentado las artes -la música como la más emblemática- por supervivir ante sociedades que no las valoran o que no priorizan el desarrollo de habilidades y destrezas en los seres humanos. "Estas luchas no se circunscriben exclusivamente a debates metodológicos, más bien las prioridades se dirigen hacia la supervivencia del arte como una materia escolar" (López, 2010, 56). Aunque actualmente la mentalidad respecto a las artes se ha visto modificada en beneficio de estas, aún es un hecho innegable que no se da el valor y la importancia suficientes a los favores que ofrece la música dentro de la educación general obligatoria.

\section{Perspectivas y desafíos de la educación musical.}

Se ha expuesto a lo largo del presente artículo perspectivas sobre la música como herramienta indispensable en el aula de clase de distintas asignaturas, tomando en cuenta la desventaja que se ha dado por la unificación de las asignaturas dentro de educación cultural y artística.

Por lo expuesto es importante considerar el contexto en el que se desarrolla el proceso educativo en cada institución puesto que no todas las instituciones ni todos los estudiantes poseen las mismas necesidades y para entender el impacto del programa de música en el contexto educativo ecuatoriano es preciso "entender a las instituciones educativas dentro de los contextos ecológicos en los cuales existen” (Abril y Abril, 2017, 31).

De igual manera Abril y Abril (2017) plantean que se debe considerar este espacio íntimo en el que se desarrolla todo el proceso educativo pues, a partir de éste se aplicarán estrategias que realmente sean efectivas a futuro, obteniendo así un ambiente escolar agradable en todos los sentidos. El contexto al que se hace referencia en este párrafo en socio-ecología es conocido como un nivel microsistema que coexiste con el "meso-sistema, exo-sistema y el marco-sistema" (32).

Recordemos que en Ecuador, la asignatura de música ha sido llevada bajo los criterios establecidos por el paradigma de la educación tradicional, incluso la modificación del currículo no ha podido realizar innovaciones ni cambios relevantes en este campo, la consideración en el perfil de salida del estudiante, ha sido nula a pesar de que la reforma educativa sugiere pensar y actuar con una mentalidad inter y multicultural. Los docentes deberían tener un refuerzo y la capacitación suficiente en torno a los beneficios que trae una educación musical organizada, planificada y evaluada.

El currículo actual exige que se cumplan estándares y si ponemos estos aprendizajes en un estudio medible complica la situación porque la música no está en base de una receta, ayuda y potencia destrezas, pero los resultados de aprendizaje son diferentes. De allí que, tomando las palabras de López (2010) "el modelo de educación fundamentada en estándares afecta negativamente a la educación musical, debido a que todo debe ser ... medible y el progreso de cada estudiante comparado con otros niños de su misma edad" (34). Aunque esta premisa se refiere a la situación educativa de Puerto Rico, se la puede tener como pauta para el contexto ecuatoriano en la medida en que establece ciertas semejanzas y una de ellas es que los docentes no están lo suficientemente preparados para impartir una educación relacionada a la elaboración y acompañamiento artístico de un ser humano.

\section{Conclusiones:}

En sentido estricto, la música es considerada como arte, como ciencia y como técnica razón por la cual su aplicación práctica permite el desarrollo cerebral y nervioso del ser humano, activa la parte cognitiva, procedimental y emocional del sujeto, pues a decir de DomingoCebrián, V. (2008) "la ejecución musical, al desarrollar las posibilidades de nuestros circuitos 

en los estudiantes

neuromusculares, permite no sólo cultivar el sistema nervioso, sino trabajar también nuestro desarrollo en general, nuestros estados afectivos, nuestra receptividad, nuestra atención, etc." (1).

Por consiguiente, la educación musical estimula todas las facultades del ser humano: abstracción, razonamiento lógico y matemático, imaginación, memoria, orden, creatividad, comunicación y perfeccionamiento de los sentidos, entre otras. Es importante valorar a la educación musical como parte fundamental en la formación integral, íntegra y globalizadora de la persona.

La música utilizada como estrategia didáctica en el aula promueve el desarrollo de habilidades y destrezas en el ser humano por ello debería ser considerada como uno de los ejes transversales para todas las asignaturas del currículo académico. La música en la educación incrementa el nivel de socialización, acerca activamente a las personas como ejecutores o como escuchas, favorece el respeto por los otros y el trabajo colaborativo.

Revisando el proceso histórico, se encuentra que fueron los pitagóricos quienes dieron a la música un valor ético y terapéutico, ellos consideraban que "la música afecta al alma y es capaz de devolverle el equilibrio perdido gracias a la dulzura de los sonidos y a la proporción matemática de sus ritmos" (Domingo-Cebrián, 2008, 1). Es decir que todos los estados de ánimo perturbados e inquietos se apaciguaban con el ritmo y la armonía de la música. El valor expresivo y emotivo de la música en sus diversos ritmos pueden influir psicológicamente en el ser humano, pueden cambiar su forma de ser, de pensar y de actuar ya que la acción estimulante o calmante de la música puede transformar al ser humano, puede contribuir para la solución de diversos problemas.

Asimismo, en la filosofía de la educación, la música contribuye para el desarrollo de la personalidad humana en la medida en que produce el enriquecimiento estético y propicia el desarrollo del optimismo y el bienestar personal. En la Grecia antigua, se valoraba a la música (junto a la gimnasia y a la poesía) como aquella que proporcionaba orden, armonía y equilibrio al ser humano. La música tiene también un valor ético-social al facilitar el dominio de uno mismo, la sensibilidad y la proyección del individuo en sociedad.

La sociedad actual signada por la ciencia, la tecnología, el consumismo y el individualismo exige la formación integral de las personas para ello es importante concentrarse no solamente por el mejoramiento de la calidad de vida sino también por la humanización, el sentido de competitividad, la formación en valores, las relaciones humanas, etc. Para cumplir con esta finalidad, la música se convierte en el vehículo ideal para lograrlo, puesto que ella se encuentra vinculada a la cultura, a los saberes, a la sociedad y a todas las manifestaciones humanas.

En fin, la música proporciona experiencias cognitivas (lenguaje y ciencia) y sensitivas (arte), participa del aporte educativo de las tres áreas del saber y es una herramienta de vital importancia para las demás asignaturas del currículo educativo por lo que se sugiere la inclusión de la asignatura de música en los planes de estudio de los todos los niveles educativos.

\section{Bibliografía}

Abril, C. y Abril, J. (2017). "Educación Musical Escolar en las Américas: Condiciones, Prácticas y Políticas desde una Perspectiva Socio-Ecológica". Revista Electrónica Complutense de Investigación Musical, 14:29-95. 
Aguilar Gordon Floralba del Rocío/ Villacís Marín Daniela Alejandra/ Narváez Flores Steven La educación musical como herramienta para el desarrollo de destrezas y habilidades en los estudiantes

Aguilar-Gordón, F. (2017). "Estrategias didácticas para desarrollar operaciones mentales en el sujeto que aprende". Revista Tópos para un debate de lo educativo, 9: 45-54, recuperado de:

http://ojs.cfe.edu.uy/index.php/rev topos/article/view/224/156

Aguilar-Gordón, F. (2016). "Implicaciones del conocimiento de los estilos de aprendizaje en el ejercicio profesional del docente universitario". Journal of Learning Styles 9 (18): 165-204.

Alvarado, R. (2013). "La música y su rol en la formación del ser humano. Universidad de chile", 1-18. Recuperado de «http://repositorio.uchile.cl/bitstream/handle/2250/122098/La_musica_y_su_rol_en_la_f ormacion_del_ser_humano.pdf;sequence=1).

Blacking, J. (2003). ¿Qué tan musical es el hombre?”. Desacatos (12): 149-162. Recuperado en 02 de marzo de 2018, «http://www.scielo.org.mx/scielo.php?script=sci_arttext\&pid=S1607050X2003000200011\&lng=es\&tlng=es0101).

Bordones, M. (2013). “Educación musical en Venezuela”. Valencia 23 (41): 119-132. 〈http://servicio.bc.uc.edu.ve/educacion/revista/n41/art09.pdf〉.

Currículo del Ecuador (2008). Recuperado de «https://educacion.gob.ec/wpcontent/uploads/downloads/2016/08/Curriculov2.pdf`.

Chao, R., Mato, M. y López, A. (2015). ¿Se trabajan de forma interdisciplinar música y matemáticas en educación infantil? Educação e Pesquisa. 42 (4): 1009-1022. 〈http://www.scielo.br/pdf/ep/v41n4/1517-9702-ep-41-4-1009.pdf〉.

Díaz, L. (2010). “Música, lenguaje y emoción: una aproximación cerebral”, Salud Mental, 33 (6): 543-541 Recuperado de «http://www.scielo.org.mx/scielo.php?script=sci_arttext\&pid=S018533252010000600009\&lng=es\&tlng=es).

Domingo-Cebrián, V. (2008). El valor de la educación musical en el desarrollo integral de la persona, en EDUCAWEB, Colegio La Salle-San José de Teruel, recuperado de: «https://www.educaweb.com/noticia/2008/02/11/valor-educacion-musical-desarrollointegral-persona-2793/>.

Duran, M. y Castell, P. (2012). “La importancia de la educación artística en la enseñanza obligatoria: la adquisición de las competencias básicas de primaria en un centro integrado de música". Revista de Educación, 357: 179-202.

Lotova, A. (2008). La educación musical en la educación infantil de España y Bulgaria: análisis comparado entre centros de Bulgaria y centros de la comunidad autónoma de Madrid. Facultad de educación. Universidad Complutense de Madrid: 3-517. 
Aguilar Gordon Floralba del Rocío/ Villacís Marín Daniela Alejandra/ Narváez Flores Steven La educación musical como herramienta para el desarrollo de destrezas y habilidades en los estudiantes

López, R. (2010). “La educación musical escolar (6-12 años) en Puerto Rico: un estudio desde la perspectiva de los maestros de música". (Tesis doctoral inédita). Universidad de Granada, 19-395. 〈https://hera.ugr.es/tesisugr/19528152.pdf〉.

Manes, F. (2015). “¿Qué hace la música a nuestro cerebro? El País. 〈https://elpais.com/elpais/2015/08/31/ciencia/1441020979_017115.html〉

Ortiz García, Martha, Vicedo Tomey, Agustín, González Jaramillo, Suleida, \& Recino Pineda, Uvaldo. (2015). "Las múltiples definiciones del término «competencia» y la aplicabilidad de su enfoque en ciencias médicas". EDUMECENTRO, 7(3): 20-31. «http://scielo.sld.cu/scielo.php?script=sci_arttext\&pid=S2077$28742015000300002 \& \operatorname{lng}=$ es\&tlng=pt .

Pérez, S. (2014). La música como herramienta para desarrollar la competencia intercultural en el aula. Perfiles educativos,36 (145): 175-187. Recuperado en 02 de marzo de 2018. «http://www.scielo.org.mx/scielo.php?script=sci_arttext\&pid=S0185$26982014000300011 \&$ Ing=es\&tlng=es .

Pérez de la Cruz, C. (2013). Educación, música y matemáticas: Un triángulo afinado en armonía. (Trabajo de fin de grado en educación primaria). Universidad de Valladolid.

Robert P. (2006). La Educación En Finlandia: Los Secretos De Un Éxito Asombroso.

Francia. Traducción por Rodríguez, M. Recuperado de 〈https://www.mineducacion.gov.co/cvn/1665/printer-121492.html〉.

Schmid, S. (2006) Competencias, habilidades cognitivas, destrezas prácticas y actitudes. Definiciones y desarrollo. 1-5. Recuperado de 〈https://rmauricioaceves.files.wordpress.com/2013/02/definicioncomphabdestrezas.pdf».

Toscano, C., Fuentes, M. (2012). “La música como herramienta facilitadora del aprendizaje del inglés como lengua extranjera. Teoría de la Educación". Revista Interuniversitaria. 24 (2): 197-213. 〈file://C:/Users/USER/Downloads/10361-37899-1-PB.pdf〉.

Vernia, A., Gustems, J. y Calderón, C. (2016). Ritmo y procesamiento temporal. Aportaciones de Jacques Dalcroze al lenguaje musical. Revista Magíster, 28 (1):1-50. http://www.elsevier.es/es-revista-magister-375-articulo-ritmo-procesamiento-temporalaportaciones-jaques-dalcroze-S0212679616300032 
Aguilar Gordon Floralba del Rocío/ Villacís Marín Daniela Alejandra/ Narváez Flores Steven La educación musical como herramienta para el desarrollo de destrezas y habilidades en los estudiantes

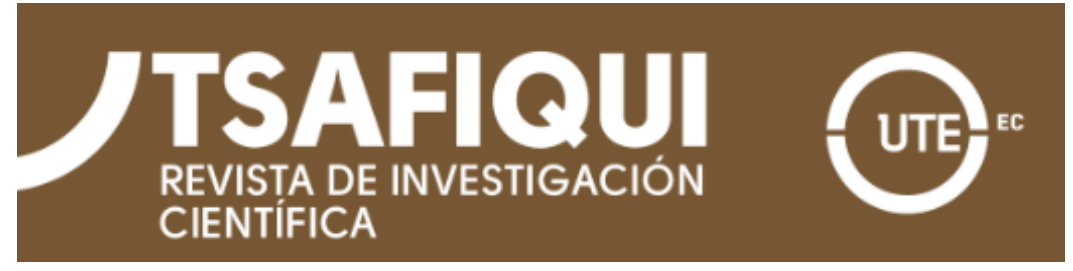

